\title{
$10 \mathcal{L}-\mathcal{R}$ duality
}

We now introduce some notation and note some simple results: these latter are valid in categories more general than the homotopy category. Given a map $f: X \rightarrow Y$, we consider classes $u$ and $\widetilde{u}$ which make the diagram commutative.

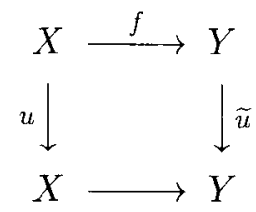

We define (cf. Rutter [1970A, §1]) the following groups which occur in the sequences used for calculations

$$
\begin{array}{r}
\mathcal{R}_{f}(X)=\left\{u \in \mathcal{E}^{*}(X): \exists \widetilde{u} \in \mathcal{E}^{*}(Y)\right\} \quad \text { and } \\
\mathcal{L}_{f}(Y)=\left\{\widetilde{u} \in \mathcal{E}^{*}(Y): \exists u \in \mathcal{E}^{*}(X)\right\}
\end{array}
$$

and their normal subgroups

$$
\begin{aligned}
\mathcal{R}_{f}^{1}(X) & =\operatorname{stab}_{f} \mathcal{E}^{*}(X)=\left\{u \in \mathcal{E}^{*}(X): \widetilde{u}=1_{Y}\right\} \quad \text { and } \\
\mathcal{L}_{f}^{1}(Y) & =\operatorname{stab}_{f} \mathcal{E}^{*}(Y)=\left\{\widetilde{u} \in \mathcal{E}^{*}(Y): u=1_{X}\right\} .
\end{aligned}
$$

These normal subgroups correspond to the indeterminacy involved in determining $u$ from $\widetilde{u}$ and vice-versa. We have the following basic result.

Lemma 10.1. (Rutter [1970A, Theorem 2.1])

$$
\mathcal{L}_{f}(Y) /_{\mathcal{L}_{f}^{1}(Y)} \cong \mathcal{R}_{f}(X) /_{\mathcal{R}_{f}^{1}(X) .}
$$

The short exact sequences determined by this isomorphism give one of the sequences which is used in the calculations. For use in the sequences relating to cellular decompositions we introduce the semigroup

$$
\mathcal{S}_{f}(X)=\left\{u \in(X, X): \exists \widetilde{u} \in \mathcal{E}^{*}(Y)\right\} .
$$

In this case we define an equivalence relation on $\mathcal{S}_{f}(X)$ by $u R u^{\prime}$ if, and only if, $f_{*}(u)=f_{*}\left(u^{\prime}\right)$, and we denote the quotient set under this relation by $f_{*}\left(\mathcal{S}_{f}(X)\right)$. Define a product on $f_{*}\left(\mathcal{S}_{f}(X)\right)$ by $f_{*}(u) \cdot f_{*}\left(u^{\prime}\right)=f_{*}\left(u u^{\prime}\right)$.

Lemma 10.2. Let $f: X \rightarrow Y$ be the inclusion of the $(n-1)$-skeleton of a path-connected $C W$-complex into the n-skeleton, then $f_{*}\left(\mathcal{S}_{f}(X)\right)$ is a group and $\mathcal{E}^{*}(Y) / \mathcal{L}_{f}^{1}(Y) \cong f_{*}\left(\mathcal{S}_{f}(X)\right)$. 